\title{
Comparative Characterization of Four Calcium-Binding EF Hand Proteins from Opisthorchis viverrini
}

\author{
Palida Emmanoch', Nanthawat Kosa', Suksiri Vichasri-Grams², Smarn Tesana ${ }^{3}$, Rudi Grams', \\ Amornrat Geadkaew-Krenc ${ }^{1, *}$ \\ ${ }^{1}$ Graduate Program in Biomedical Sciences, Faculty of Allied Health Sciences, Thammasat University, Pathumthani 12120, Thailand; ${ }^{2}$ Department of \\ Biology, Faculty of Science, Mahidol University, Bangkok 10400, Thailand; ${ }^{3}$ Food-borne Parasite Research Group, Department of Parasitology, \\ Faculty of Medicine, Khon Kaen, 40002, Thailand
}

\begin{abstract}
Four isoforms of calcium binding proteins containing $2 \mathrm{EF}$ hand motifs and a dynein light chain-like domain in the human liver fluke Opisthorchis viverrini, namely OvCaBP1, 2, 3, and 4, were characterized. They had molecular weights of 22.7, 21.6, 23.7, and $22.5 \mathrm{kDa}$, respectively and showed $37.2-42.1 \%$ sequence identity to CaBP22.8 of O. viverrini. All were detected in 2- and 4-week-old immature and mature parasites. Additionally, OvCaBP4 was found in newly excysted juveniles. Polyclonal antibodies against each isoform were generated to detect the native proteins in parasite extracts by Western blot analysis. All OvCaBPs were detected in soluble and insoluble crude worm extracts and in the excretory-secretory product, at approximate sizes of 21-23 kDa. The ion-binding properties of the proteins were analyzed by mobility shift assays with the divalent cations $\mathrm{Ca}^{2+}, \mathrm{Mg}^{2+}, \mathrm{Zn}^{2+}$, and $\mathrm{Cu}^{2+}$. All OvCaBPs showed mobility shifts with $\mathrm{Ca}^{2+}$ and $\mathrm{Zn}^{2+}$. OvCaBP1 showed also positive results with $\mathrm{Mg}^{2+}$ and $\mathrm{Cu}^{2+}$. As tegumental proteins, OvCaBP1, 2, and 3 are interesting drug targets for the treatment of opisthorchiasis.
\end{abstract}

Key words: Opisthorchis viverrini, Platyhelminthes, EF hand motif, calcium-binding, dynein light chain, tegument

Opisthorchis viverrini is an important human parasite and chronic infection may lead to cholangiocarcinoma (CCA) [1]. The highest prevalence of the parasite infection is in the Lower Mekong basin, including Thailand, Laos, and Vietnam [2,3]. Praziquantel is the first choice of drug for opisthorchiasis and other foodborne trematodiases. Praziquantel-resistance has been reported in Schistosoma mansoni and possibly in S. japonicum [4], but as yet there has been no report of it in O. viverrini [5]. The mechanism of praziquantel against helminths is still unclear [6], but it involves tegumental damage caused by vacuolization rupture $[7,8]$. Tegumental antigens have been intensively studied for the development of drug targets and diagnostic tools. Calcium binding proteins (CaBPs) comprising 2 EF hand motifs and a dynein light chain (DLC) like domain have been identified and characterized in trematodes including Fasciola sp., Schistosoma sp., Clonorchis sinensis, and O. viverrini. Many of these CaBPs are located at the tegument layer of

- Received 19 October 2017, revised 19 February 2018, accepted 19 February 2018.

*Corresponding author (amornrut_gead@hotmail.com)

(c) 2018, Korean Society for Parasitology and Tropical Medicine

This is an Open Access article distributed under the terms of the Creative Commons Attribution Non-Commercial License (http://creativecommons.org/licenses/by-nc/4.0) which permits unrestricted non-commercial use, distribution, and reproduction in any

medium, provided the original work is properly cited. the parasites. The schistosome CaBPs, termed tegumental allergen-like proteins (TALs) [9], and Fasciola gigantica CaBPs [10], can strongly induce an IgE immune response in the hosts. However, the cellular mechanisms in which this protein family is involved remain unclear. In vitro studies showed interaction of $S$. mansoni and $F$. hepatica CaBPs $[11,12]$ with drugs including praziquantel suggesting that these proteins merit further investigations. In the following, we briefly describe a basic molecular analysis of $4 \mathrm{CaPBs}$ from O. viverrini, which has been done in preparation for research on their suitability as drug targets.

All animal experiments in this study were approved by the Thammasat University Animal Ethics Committee (project no. 014/2557, 28 October 2014). Syrian golden hamsters (Mesocricetus auratus) were infected with $O$. viverrini metacercariae collected from naturally infected fish to obtain immature and mature parasites. Female ICR mice were used for immunization to generate anti-recombinant OvCaBP antisera. All experimental details can be found in the Supplementary Text and Supplementary Tables 1-3. The cDNAs encoding 4 O. viverrini calcium binding proteins, i.e., OvCaBP1, 2, 3, and 4 (numbers solely appended for discrimination), and calcium binding experimentally verified as described below (GenBank no. MF 
767953-MF767956), were isolated by reverse transcriptase PCR (RT-PCR) from adult stage total RNA extracted in TRIzol using primer pairs (1) ggatccATGACACAACAAGCAGCACA, aagcttCTATGCGCGGTTAGTACG; (2) ggatccATGGAAGGCATTGAATCAATG, aagcttTTAACACTGAGGGGTGCG; (3) catatgGCACAGGTTCAAACG, ctcgagCGTCCGGTTCGTACGCCA; (4) ggatccATGGGTGAACAAGGATCG, aagcttTTAGTTGATGGTGGTACG) and inserted into pGEM-T Easy. They were selected from 19 family members that were identified by BLAST searches in the genome/transcriptome data of $O$. viverrini and C. sinensis [13-16]. Analysis of the deduced amino acid sequences showed that the 4 OvCaBPs had $37.2-42.1 \%$ sequence identity with $\mathrm{O}$. viverrini calcium binding protein $\mathrm{Ov}$ CaBP22.8 which until now had been the only reported family member in this parasite [17]. OvCaBP22.8 was identified by immunoscreening with the serum of a CCA patient, interestingly it was not detected in the parasite tegument but in the digestive tract and parenchyma. Predicted proteins GAA34310, GAA47752, and GAA37705 in the draft genome of C. sinensis [4] and characterized tegumental protein CsTegu21.6 [18] showed very high sequence identity (91-98\% by NCBIBLASTP) with OvCaBP1, 2, 3, and 4, respectively, and obviously represent the orthologs in this closely related species.
The sequence of each OvCaBP contains a pair of $\mathrm{EF}$ hand motifs in the N-terminal half, and a DLC-like domain in the Cterminal half (Fig. 1), as predicted by PROSITE and InterPro.

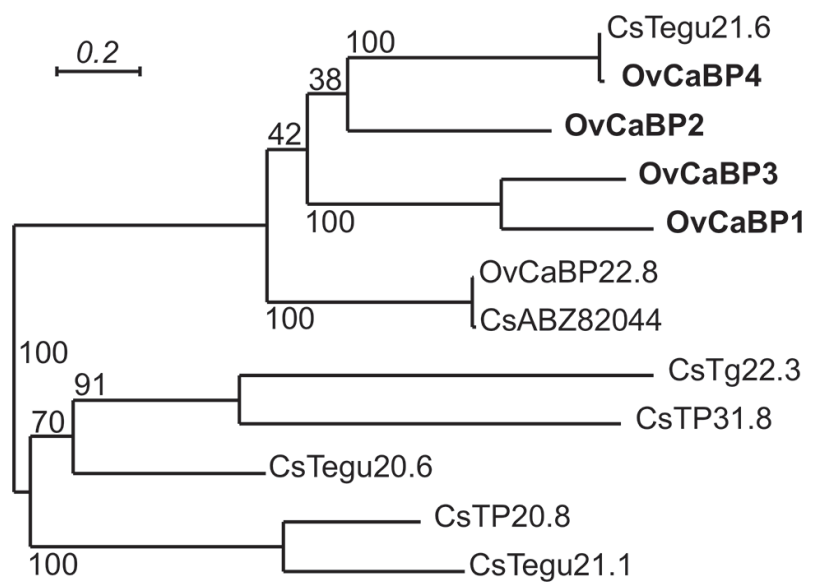

Fig. 2. Phylogenetic tree based on maximum-likelihood analysis of characterized O. viverrini/C. sinensis CaBPs. The described OvCaBP1-4 are indicated in bold. CsTegu21.6, AEl69651, [18]; OvCaBP22.8, XP_009173200, [17]; CsABZ82044, ABZ82044, [27]; CsTg22.3, ABK60085, [28]; CsTP31.8, ABK60086, [29]; CsTegu20.6, GAA49981, [25]; CsTP20.8, ABC47326, [30]; CsTegu21.1, ADZ13689, [31]. The bootstrap support values are shown at the nodes, this is an unrooted tree, log likelihood: -3549.0 .

\begin{tabular}{|c|c|c|}
\hline & EF hand I & \\
\hline & $\nabla \nabla \nabla \nabla \nabla \nabla$ & \\
\hline & MTQQAAQSNVVEELMKCFMLLDVNNDGFVSREELVDFCQKHQLNPA & \\
\hline & MEGI.SmiDW. lEm $\cdot \mathrm{K} \cdot \mathrm{E} \cdot \mathrm{GV} \cdot \mathrm{DCK} \cdot \cdot \mathrm{E}$ & \\
\hline & AQVQTPKPHKTELHTT $\cdot \mathrm{QlmMF} \cdot \mathrm{mKl} \cdot \mathrm{K} \cdot \mathrm{N} \cdot \mathrm{GV} \cdot \mathrm{DRN} \cdot \cdot \mathrm{LDFCQKI}$ & \\
\hline & $\mathrm{MGEQGSDM} \cdot \mathrm{KmiEM} \cdot \mathrm{lGm} \cdot \mathrm{K} \cdot \mathrm{D} \cdot \mathrm{GF} \cdot \mathrm{DLG} \cdot \mathrm{F}$ & \\
\hline 2.8 & $=1, T \cdot R \cdot F Y \cdot D R B$, & \\
\hline & EF hand II & \\
\hline & $\begin{array}{llllll}\boldsymbol{\nabla} & \boldsymbol{\nabla} & \boldsymbol{\nabla} & \boldsymbol{\nabla} & \boldsymbol{\nabla}\end{array}$ & \\
\hline$\checkmark C a B P 1$ & FDVDADGKISFEEYARGLGLTIQEVQQEKKE & \\
\hline & $f \cdot K N D \cdot N K \cdot t L A \cdot f C$ & \\
\hline & $\cdot G K \cdot t L N \cdot y$ & 104 \\
\hline & $\cdot T N K \cdot G K \cdot S L D \cdot f C$ & \\
\hline $\mathrm{CaBP} 22.8$ & EvSHwlDky $\cdot \mathrm{LNG} \cdot \mathrm{GN} \cdot \mathrm{tLD} \cdot \mathrm{fCHa} \cdots \mathrm{KCE}$ emRI $\cdot$ rYERQ - - RERDGFAKV & \\
\hline & DLC like domain & \\
\hline & LQFEDVEILSTSMTWSKQETIINKFKELVGGGDPNEEA & \\
\hline & $\mathrm{fMD} \cdot \mathrm{CKTHGTDSNGm}$ & 14 \\
\hline & KA V VGSGFPTFFKM & \\
\hline & - fIE AKEVSSDPHKMNQ. & 14 \\
\hline OvCaBP22.8 & l-NPDVSI IAst - sLD - VD - TN · fVE - LKETSGRPED INE - AKNLKDy IDK & \\
\hline & & \\
\hline & & \\
\hline & $\cdot \mathrm{kv} \cdot \mathrm{CV}$ ilT $\cdot \mathrm{f} \cdot \mathrm{mRf} \cdot \mathrm{YE} \cdot \mathrm{m} \cdot \mathrm{i} \cdot \mathrm{RHKNKFVVLA} \cdots \mathrm{N}$ & \\
\hline & $\cdots r V \cdots V i v l A \cdots y \cdot i N y \cdot H A \cdots l \cdot m \cdot Q Y$ GPYiCIV $\cdots$ TIN & \\
\hline OvCaBP & $\mathrm{Q} \cdots \mathrm{rv} \cdots \mathrm{TVlvA} \cdot \mathrm{y} \cdot \mathrm{mKf} \cdot \mathrm{HE} \cdots \mathrm{m} \cdot \mathrm{l} \cdot \mathrm{KC}$ GPHiCLV$\cdots \mathrm{PCIERDSFN}$ & \\
\hline
\end{tabular}

Fig. 1. Multiple sequence alignment of OvCaBP1-4 and OvCaBP22.8. The $2 \mathrm{EF}$ hand motifs and single dynein light chain like domain are indicated. The 6 residues in each EF hand motif making contact to calcium are indicated by triangles ( $\mathbf{\nabla})$. OvCaBP1 is used as reference sequence. Positions with all identical residues are indicated as dots $(\cdot)$, positions with all similar residues are shown in lower letters. Gaps introduced for alignment are indicated by dashes (-). 
The deduced numbers of amino acids of OvCaBP1-4 are 195, 184,201 , and 200 residues, with molecular weights of 22.7, 21.6, 23.7, and $22.5 \mathrm{kDa}$ as calculated in EMBOSS pepstats [19], respectively. In pairwise comparison OvCaBP1 and OvCaBP3 show the highest sequence identity at $52.2 \%$, OvCaBP3 and OvCaBP4 the lowest at 32.6\% (see Supplementary Tables and Figures for details, EMBOSS needle). Sequence conservation was in general higher in the calcium-binding regions of the EF hand motifs and the C-terminal half of the DLC-like domain (Fig. 1). While the orthologs in O. viverrini and C. sinensis were highly conserved at $>90 \%$ identity, this was most often not the case when comparing paralogous family members and was evident by low bootstrap support values in the phylogenetic tree (Fig. 2; Supplementary Fig. 1) constructed in PhyML 3.0 [20]. OvCaBP1-3 represent previously uncharacterized CaBP in both species.

Total RNA was extracted from newly excysted juveniles (NEJ) and 2-, 4-, and 8-week-old parasites by using TRIzol. The transcripts of OvCaBP1-4 were amplified by RT-PCR using the mentioned primers for each isoform and resolved by agarose gel electrophoresis. RNA products were found in 2-week-old juveniles through adult stage for all 4 genes. In addition, OvCaBP4 transcripts were faintly detected in NEJ (Fig. 3). Inferring from the results, the proteins are not important in dor- mant metacercariae and for excystation but are used in larger amounts in the parasite growth phase.

The OvCaBP cDNAs were subcloned into either prokaryotic expression vector pQE30 (N-terminal His-tag) or pET21b (Cterminal His-Tag, OvCaBP3) and recombinant OvCaBPs (rOvCaBPs) were expressed in soluble form in Escherichia coli and purified by metal affinity chromatography through the introduced histidine-tags (Supplementary Fig. 2). The metal ionbinding properties of rOvCaBPs were analyzed by mobility

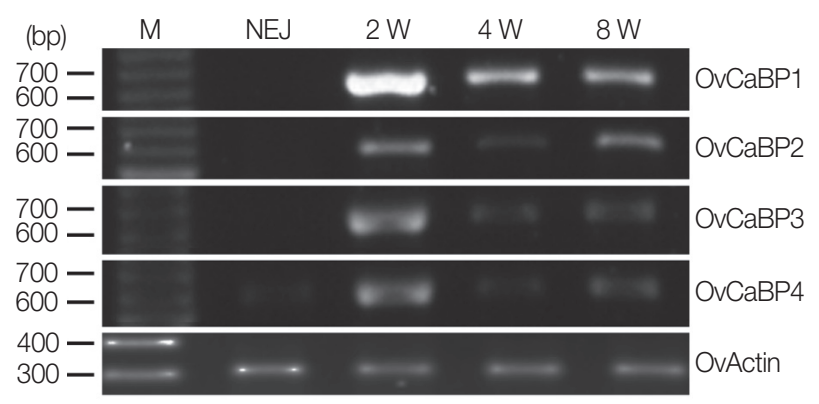

Fig. 3. Stage-specific amplification of OvCaBPs transcripts by reverse transcriptase PCR. The total RNA of newly excysted juveniles (NEJ), 2-week juveniles (2 W), 4-week juveniles (4 W), and 8-week adult (8 W) O. viverrini were extracted in TRIzol and used as templates for RT-PCR with specific primers for each isoform. OvActin was used as standard. Lane M, 100 bp DNA ladder.
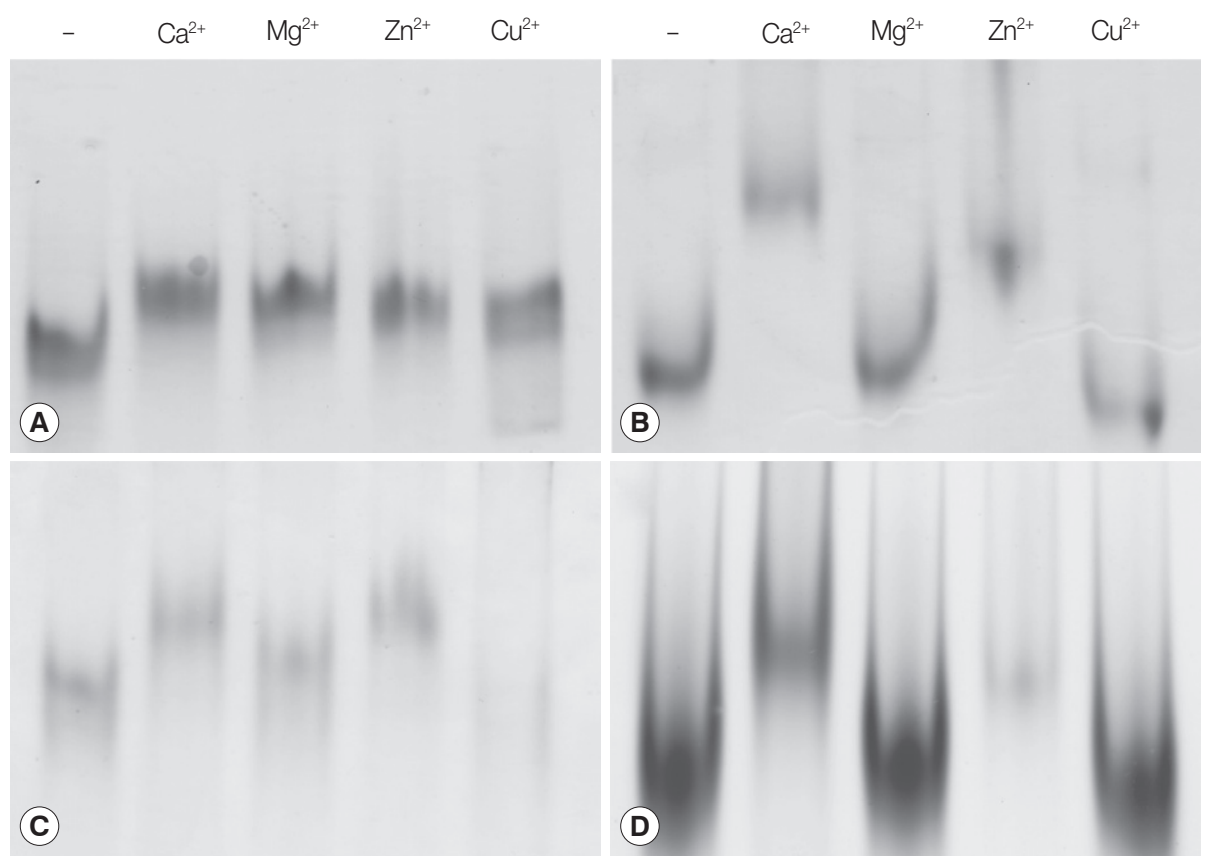

Fig. 4. Determination of ion-binding properties by mobility shift assays in non-denaturing gels. Five micrograms of rOvCaBP (A-D) were pre-incubated with $5 \mathrm{mM}$ EDTA and post-incubated with $25 \mathrm{mM} \mathrm{CaCl}_{2}, \mathrm{MgCl}_{2}, \mathrm{ZnSO}_{4}$, and $\mathrm{CuSO}_{4}$. Minus (-) symbols indicate proteins only incubated with $5 \mathrm{mM}$ EDTA. 


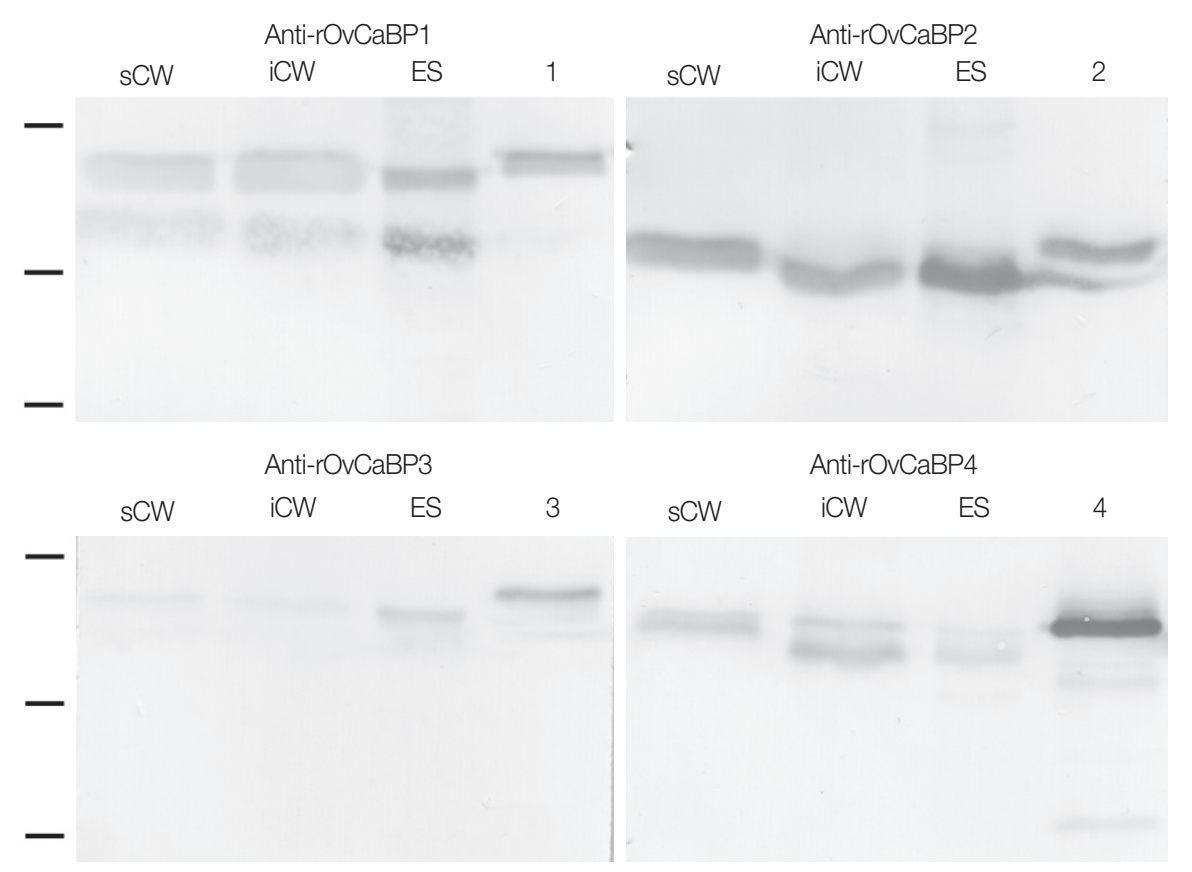

Fig. 5. Western blot analysis of mature O. viverrini crude worm extracts (CW), ES product (ES), and recombinant OvCaBP1-4 (1, 2, 3, and 4) with mouse anti-rOvCaBP1-4 antisera at dilution 1:2,000. sCW, $20 \mu \mathrm{g}$ soluble CW; iCW, $20 \mu \mathrm{g}$ insoluble CW; ES, $20 \mu \mathrm{g}$ ES product; lanes 1, 2, 3, and 4, $100 \mathrm{ng}$ of rOvCaBP-1, -2, -3, and -4, respectively. Positions of 31.0, 21.5, and 14.4 kDa protein standards are indicated on the left.

shift assays in non-denaturing PAGE (Fig. 4; Supplementary Fig. 3). $\mathrm{Ca}^{2+}$ and $\mathrm{Zn}^{2+}$ were bound by all rOvCaBPs. $\mathrm{Mg}^{2+}$ was bound by rOvCaBP1 and 3, $\mathrm{Cu}^{2+}$ only by rOvCaBP1. The metal ion binding properties of CsTegu21.6 [18], the ortholog of OvCaBP4 in C. sinensis were not reported. Binding with other divalent cations has been described for Sm20.6, Sm21.7, Sm20.8, FhCaBP2 and FhCaBP3 [11,12,21]. The binding of $\mathrm{Ca}^{2+}$ and other metal ions has been shown to take place at the helix-loop-helix structure of the EF hand motifs [22]. Binding with metal ions might have an effect on protein structure and therefore affect protein function.

The purified rOvCaBPs were used to produce antisera in mice ( 3 mice per antigen) and these antisera were then used for western detection of the recombinant and native antigens. The antigens were resolved by SDS-PAGE and transferred onto nitrocellulose membranes by semidry blotting. The antisera detected recombinant and native antigens at their expected molecular weights in soluble and insoluble crude worm extracts (CW) and ES product (Fig. 5). Presence of OvCaBPs in the ES product suggests their release through non-classical secretion as they lack signal peptides. The antisera showed some cross-reactivity, potentially to other OvCaBP isoforms. Varying cross-reactivity was confirmed by testing each antiserum against the 4 rOvCaBPs in western blots (Supplementary Fig. 4) and has also been reported for CaBPs in F. gigantica [10]. This may be due to conserved immunodominant epitopes in their EF hand motifs as these could induce strong IgE immune responses in S. haematobium [23], S. mansoni [24], and F. gigantica [10]. OvCaBPs were detected immunohistochemically in the tegument and tegumental cell bodies of adult $O$. viverrini (Supplementary Fig. 5).

In conclusion, the 4 described $O$. viverrini proteins are typical members of a large Platyhelminthes-specific subfamily in the family of calcium binding EF hand carrying proteins with the characteristic combination of $2 \mathrm{EF}$ hand motifs and a single DLC like domain. The presence of all OvCaBPs in the ES product of the mature parasite was noted and preliminary data from infected hamsters suggests that at least OvCaBP2 is stimulating an immune response (data not shown). Despite modest sequence identity cross reactivity of antisera was observed which will impede application of OvCaBPs as diagnosis tools. On the other hand, the observed sequence divergences should result in isoform-specific surface structure/charge properties and future studies should focus on the drug binding potentials of the different isoforms. 


\section{ACKNOWLEDGMENT}

Nucleotide sequence data reported in this paper are available in the EMBL, GenBank, and DDJB databases under the accession nos. MF767953, MF767954, MF767955, and MF 767956. This research was financially supported by a grant through Thammasat University, contract no: 35/2559 and 44/2560.

\section{CONFLICT OF INTEREST}

We declare that we have no conflict of interest related to this work.

\section{REFERENCES}

1. Sripa B, Brindley PJ, Mulvenna J, Laha T, Smout MJ, Mairiang E, Bethony JM, Loukas A. The tumorigenic liver fluke Opisthorchis viverrini - multiple pathways to cancer. Trends Parasitol 2012; 28: 395-407.

2. Sithithaworn P, Haswell-Elkins M. Epidemiology of Opisthorchis viverrini. Acta Trop 2003; 88: 187-194.

3. Sayasone S, Odermatt P, Phoumindr N, Vongsaravane X, Sensombath V, Phetsouvanh R, Choulamany X, Strobel M. Epidemiology of Opisthorchis viverrini in a rural district of southern Lao PDR. Trans R Soc Trop Med Hyg 2007; 101: 40-47.

4. Wang W, Wang L, Liang YS. Susceptibility or resistance of praziquantel in human schistosomiasis: a review. Parasitol Res 2012; 111: 1871-1877.

5. Keiser J, Utzinger J. The drugs we have and the drugs we need against major helminth infections. Adv Parasitol 2010; 73: 197230.

6. Cioli D, Pica-Mattoccia L, Basso A, Guidi A. Schistosomiasis control: praziquantel forever? Mol Biochem Parasitol 2014; 195 : 23-29.

7. Pax R, Bennett JL, Fetterer R. A benzodiazepine derivative and praziquantel: effects on musculature of Schistosoma mansoni and Schistosoma japonicum. Naunyn Schmiedebergs Arch Pharmacol 1978; 304: 309-315.

8. Mehlhorn H, Kojima S, Rim HJ, Ruenwongsa P, Andrews P, Thomas H, Bunnag B. Ultrastructural investigations on the effects of praziquantel on human trematodes from Asia: Clonorchis sinensis, Metagonimus yokogawai, Opisthorchis viverrini, Paragonimus westermani and Schistosoma japonicum. Arzneimittelforschung 1983; 33: 91-98.

9. Jeffs SA, Hagan P, Allen R, Correa-Oliveira R, Smithers SR, Simpson AJ. Molecular cloning and characterisation of the 22-kilodalton adult Schistosoma mansoni antigen recognised by antibodies from mice protectively vaccinated with isolated tegumental surface membranes. Mol Biochem Parasitol 1991; 46: 159-167.

10. Subpipattana P, Grams R, Vichasri-Grams S. Analysis of a calci- um-binding EF-hand protein family in Fasciola gigantica. Exp Parasitol 2012; 130: 364-373.

11. Banford S, Drysdale O, Hoey EM, Trudgett A, Timson DJ. FhCaBP3: a Fasciola hepatica calcium binding protein with EF-hand and dynein light chain domains. Biochimie 2013; 95: 751-758.

12. Thomas CM, Fitzsimmons CM, Dunne DW, Timson DJ. Comparative biochemical analysis of three members of the Schistosoma mansoni TAL family: differences in ion and drug binding properties. Biochimie 2015; 108: 40-47.

13. Young ND, Campbell BE, Hall RS, Jex AR, Cantacessi C, Laha T, Sohn WM, Sripa B, Loukas A, Brindley PJ, Gasser RB. Unlocking the transcriptomes of two carcinogenic parasites, Clonorchis sinensis and Opisthorchis viverrini. PLoS Negl Trop Dis 2010; 4: e719.

14. Wang X, Chen W, Huang Y, Sun J, Men J, Liu H, Luo F, Guo L, Lv X, Deng C, Zhou C, Fan Y, Li X, Huang L, Hu Y, Liang C, Hu X, $\mathrm{Xu}$ J, Yu X. The draft genome of the carcinogenic human liver fluke Clonorchis sinensis. Genome Biol 2011; 12: R107.

15. Huang Y, Chen W, Wang X, Liu H, Chen Y, Guo L, Luo F, Sun J, Mao Q, Liang P, Xie Z, Zhou C, Tian Y, Lv X, Huang L, Zhou J, Hu Y, Li R, Zhang F, Lei H, Li W, Hu X, Liang C, Xu J, Li X, Yu X. The carcinogenic liver fluke, Clonorchis sinensis: new assembly, reannotation and analysis of the genome and characterization of tissue transcriptomes. PLoS One 2013; 8: e54732.

16. Young ND, Nagarajan N, Lin SJ, Korhonen PK, Jex AR, Hall RS, Safavi-Hemami H, Kaewkong W, Bertrand D, Gao S, Seet Q, Wongkham S, Teh BT, Wongkham C, Intapan PM, Maleewong W, Yang X, Hu M, Wang Z, Hofmann A, Sternberg PW, Tan P, Wang J, Gasser RB. The Opisthorchis viverrini genome provides insights into life in the bile duct. Nat Commun 2014; 5: 4378.

17. Senawong G, Laha T, Loukas A, Brindley PJ, Sripa B. Cloning, expression, and characterization of a novel Opisthorchis viverrini calcium-binding EF-hand protein. Parasitol Int 2012; 61: 94-100.

18. Kim YJ, Yoo WG, Lee MR, Kim DW, Lee WJ, Kang JM, Na BK, Ju JW. Identification and characterization of a novel 21.6-kDa tegumental protein from Clonorchis sinensis. Parasitol Res 2012; 110: 2061-2066.

19. Rice P, Longden I, Bleasby A. EMBOSS: the European Molecular Biology Open Software Suite. Trends Genet 2000; 16: 276-277.

20. Guindon S, Dufayard JF, Lefort V, Anisimova M, Hordijk W, Gascuel O. New algorithms and methods to estimate maximumlikelihood phylogenies: assessing the performance of PhyML 3.0. Syst Biol 2010; 59: 307-321.

21. Thomas CM, Timson DJ. FhCaBP2: a Fasciola hepatica calciumbinding protein with EF-hand and dynein light chain domains. Parasitology 2015; 142: 1375-1386.

22. Gifford JL, Walsh MP, Vogel HJ. Structures and metal-ion-binding properties of the Ca2+-binding helix-loop-helix EF-hand motifs. Biochem J 2007; 405: 199-221.

23. Fitzsimmons CM, Stewart TJ, Hoffmann KF, Grogan JL, Yazdanbakhsh M, Dunne DW. Human IgE response to the Schistosoma haematobium $22.6 \mathrm{kDa}$ antigen. Parasite Immunol 2004; 26: 371376. 
24. Fitzsimmons CM, Jones FM, Stearn A, Chalmers IW, Hoffmann KF, Wawrzyniak J, Wilson S, Kabatereine NB, Dunne DW. The Schistosoma mansoni tegumental-allergen-like (TAL) protein family: influence of developmental expression on human IgE responses. PLoS Negl Trop Dis 2012; 6: e1593.

25. Kim YJ, Yoo WG, Lee MR, Kang JM, Na BK, Cho SH, Park MY, Ju JW. Molecular and structural characterization of the tegumental 20.6-kDa protein in Clonorchis sinensis as a potential druggable target. Int J Mol Sci 2017; 18: 557.

26. Kim TI, Cho PY, Song KJ, Li S, Hong SJ, Park SW, Chai JY, Shin EH. Gene expression of Clonorchis sinensis metacercaria induced by gamma irradiation. Parasitol Res 2008; 102: 1143-1150.

27. Zhou Z, Xia H, Hu X, Huang Y, Ma C, Chen X, Hu F, Xu J, Lu F, Wu Z, Yu X. Immunogenicity of recombinant Bacillus subtilis spores expressing Clonorchis sinensis tegumental protein. Parasi- tol Res 2008; 102: 293-297

28. Huang Y, Zhou Z, Hu X, Wei Q, Xu J, Wu Z, Yu X. A novel tegumental protein $31.8 \mathrm{kDa}$ of Clonorchis sinensis: sequence analysis, expression, and immunolocalization. Parasitol Res 2007; 102: 77-81.

29. Zhou Z, Hu X, Huang Y, Hu H, Ma C, Chen X, Hu F, Xu J, Lu F, Wu Z, Yu X. Molecular cloning and identification of a novel Clonorchis sinensis gene encoding a tegumental protein. Parasitol Res 2007; 101: 737-742.

30. Chen J, Xu H, Zhang Z, Zeng S, Gan W, Yu X, Hu X. Cloning and expression of 21.1-kDa tegumental protein of Clonorchis sinensis and human antibody response to it as a trematode-nematode pan-specific serodiagnosis antigen. Parasitol Res 2011; 108: 161-168. 


\section{SUPPLEMENTARY TEXT ADDITIONAL MATERIALS AND METHODS}

\section{Parasites and experimental animals}

Naturally infected cyprinoid fish were collected in northeast Thailand for isolation of metacercariae as described by Srisawangwong et al. [1]. To prepare newly excysted juveniles (NEJ), around 1,500 metacercariae were excysted by alcoholenhancement [2]. Adults, 4- and 2-week-old juveniles were obtained by infection of Syrian golden hamsters (Mesocricetus auratus) with metacercariae as described by Geadkaew et al. [3]. All parasites were kept in liquid nitrogen until use. All animals experiments in this study were approved by the Thammasat University Animal Ethics Committees (Project No. 014/2557, 28 October 2014). Animals were maintained under strict hygienic conventional conditions on corncob bedding (B\&C Pulaski Corporation Ltd., Bangkok Thailand) in solid bottom cages (Tecniplast, Milan, Italy) for group housing (3 mice per cage) at temperature of $22 \pm 1^{\circ} \mathrm{C}$, humidity of $30-70 \%, 10-15$ air change per hr and 12:12 hr light/dark cycle controlled automatically. Animals were fed ad libitum with commercial diet (Charoen Pokphand, Bangkok, Thailand) and RO filtered drinking water in bottles with sipper tube (Tecniplast).

\section{Molecular cloning and sequence analysis}

NCBI-BLAST screens of Opisthorchis viverrini and Clonorchis sinensis [4-7] protein/nucleotide data were performed to iden- tify genes that encode calcium-binding proteins containing EF hand motifs and dynein light chain-like domain (CaBPs). Four undescribed genes of $\mathrm{O}$. viverrini were selected for molecular analysis and their complete coding sequences (CDS) were cloned from adult stage total RNA by reverse transcriptase PCR with the primers detailed in Table 1 . The cDNAs were inserted into pGEM-T Easy (Promega, Wisconsin) by ligation and sequenced (SolGent, Daejeon, Korea). Standard sequence analysis was performed using EMBOSS version 6.5 [8] including pairwise alignment (EMBOSS needle with parameters: EBLOSUM62, gap penalty 10.0, extend penalty 1.0 ). Phylogenetic analysis was performed starting with the ETE 3 metaligner workflow [9] that comprises Clustal Omega, MAAFT, MUSCLE, and M-Coffee [10-13] trimAl 1.4 [14] with the gappyout setting. Finally, PhyML 3.0 [15] was used to construct a maximum likelihood phylogeny using the LG model and 100 bootstraps.

\section{Reverse transcriptase PCR analysis}

Total RNA was isolated with TRIzol (Ambion, California, USA) from newly excysted juveniles (NEJ), 2-and 4-week-old juveniles and mature parasites. The extracted RNAs were treated with DNase I (Promega, Madison, Wisconsin, USA) and 100 ng of each treated RNA sample was reverse transcribed (RevertAid, Thermo Fisher Scientific, Waltham, Massachusetts, USA) to CDNAs by using specific reverse primers (Table 1) for each OvCaBP. The following standard PCR was performed by

Table 1. Primers and introduced restriction sites used for cloning the complete coding sequence of OvCaBP1-4

\begin{tabular}{|c|c|c|c|}
\hline OvCaBP & Primer sequence ${ }^{a}$ & Restriction site & Size (bp) \\
\hline 1 & $\begin{array}{l}\text { Fwd: ggatccATGACACAACAAGCAGCACA } \\
\text { Rev: aagcttCTATGCGCGGTTAGTACG }\end{array}$ & $\begin{array}{l}\text { BamH I } \\
\text { Hind II }\end{array}$ & 588 \\
\hline 2 & $\begin{array}{l}\text { Fwd: ggatccATGGAAGGCATTGAATCAATG } \\
\text { Rev: aagcttTTAACACTGAGGGGTGCG }\end{array}$ & $\begin{array}{l}\text { BamH I } \\
\text { Hind III }\end{array}$ & 555 \\
\hline 3 & $\begin{array}{l}\text { Fwd: catatgGCACAGGTTCAAACG } \\
\text { Rev: ctcgagCGTCCGGTTCGTACGCCA }\end{array}$ & $\begin{array}{l}\text { Nde I } \\
\text { Xho I }\end{array}$ & 606 \\
\hline 4 & $\begin{array}{l}\text { Fwd: ggatccATGGGTGAACAAGGATCG } \\
\text { Rev: aagcttTAGTTGATGGTGGTACG }\end{array}$ & $\begin{array}{l}\text { BamH I } \\
\text { Hind III }\end{array}$ & 597 \\
\hline
\end{tabular}

arestriction sites indicated by underlined small letters.

Table 2. Amino acid identity (bold) and similarity values (\%) of OvCaBP1-4 and OvCaBP22.8

\begin{tabular}{|c|c|c|c|c|c|}
\hline & OvCaBP1 & OvCaBP2 & OvCaBP3 & OvCaBP4 & OvCaBP22.8 \\
\hline OvCaBP1 & - & 37.9 & 52.2 & 37.4 & 37.2 \\
\hline OvCaBP2 & 57.4 & - & 35.8 & 41.9 & 42.1 \\
\hline OvCaBP3 & 71.1 & 63.2 & - & 32.6 & 38.2 \\
\hline OvCaBP4 & 60.7 & 58.6 & 55.8 & - & 41.2 \\
\hline OvCaBP22.8 & 56.0 & 61.5 & 58.5 & 58.8 & - \\
\hline
\end{tabular}


Table 3. Ion-binding patterns of rOvCaBP1-4 with $\mathrm{Ca}^{2+}, \mathrm{Mg}^{2+}$, $\mathrm{Zn}^{2+}$ and $\mathrm{Cu}^{2+}$ by mobility shift assay

\begin{tabular}{lcccc} 
& $\mathrm{Ca}^{2+}$ & $\mathrm{Mg}^{2+}$ & $\mathrm{Zn}^{2+}$ & $\mathrm{Cu}^{2+}$ \\
\hline OvCaBP1 & + & + & + & + \\
OvCaBP2 & + & - & + & - \\
OvCaBP3 & + & - & + & - \\
OvCaBP4 & + & - & + & - \\
\hline
\end{tabular}

+ , bound; -, not bound.

using Taq DNA polymerase (Thermo Fisher Scientific) and specific primers for each isoform as shown in Table 1. The RTPCR products were analyzed by $1 \%$ agarose gel electrophoresis and subsequently inserted into pGEM T- Easy by ligation.

\section{Expression and purification of rOvCaBPs}

Sequence-verified OvCaBP cDNAs were subcloned from pGEM T-Easy into the expression vectors pQE30 (for OvCaBP1, 2, and 4) or pET21b (for OvCaBP3). The expression strains Escherichia coli M15 (for pQE30) and E. coli BL21 (for pET21b) were transformed with the recombinant vectors. Following bacterial culture and induction with $1 \mathrm{mM}$ IPTG the expressed soluble recombinant proteins were purified under native conditions by Ni-NTA affinity chromatography (QIAGEN, Hilden, Germany). The purified recombinant proteins were dialyzed against different buffers depending on their solubility. OvCaBP1: $10 \mathrm{mM}$ PBS, pH 7.2; OvCaBP2 and OvCaBP3: 50 mM Tris-HCl, pH 8.0; OvCaBP4: elution buffer. The purified soluble OvCaBPs were used for functional analysis and antibody production.

\section{Preparation of crude worm extracts (CWEs) and excretory secretory product (ES product) from adult O. viverrini}

Adult $O$. viverrini worms were homogenized in lysis buffer (150 mM NaCl, 10 mM Tris-HCl pH 7.2, 1 mM EDTA pH 8.0, $1 \mathrm{mM}$ PMSF and $0.5 \%$ [v/v] Triton-X 100) by using an UltraTurrax T25 tissue homogenizer (IKA, Staufen, Germany). The homogenate was rotated at $4^{\circ} \mathrm{C}$ for $1 \mathrm{hr}$ and centrifuged at $12,000 \times \mathrm{g}$ and $4^{\circ} \mathrm{C}$ for $15 \mathrm{~min}$. The supernatant was collected as soluble CWE and the pellet was solubilized in $50 \mathrm{mM}$ Tris$\mathrm{HCl}, \mathrm{pH} 8.0$ and $3 \% \mathrm{SDS}$ at $37^{\circ} \mathrm{C}$ for $1 \mathrm{hr}$. The lysate was centrifuged at $12,000 \times \mathrm{g}$ for $15 \mathrm{~min}$ and the solubilized CWE was collected. To prepare ES product, fresh adult parasites were washed several times with $0.85 \% \mathrm{NaCl}$ and then incubated in $0.01 \mathrm{M}$ PBS, $\mathrm{pH} 7.2$ in $5 \% \mathrm{CO}$ at $37^{\circ} \mathrm{C}$ for $12 \mathrm{hr}$. The buffer was collected and centrifuged at $5,000 \times \mathrm{g}$ at $4^{\circ} \mathrm{C}$ for $20 \mathrm{~min}$ to eliminate parasite eggs and insoluble material. The supernatant was concentrated using a centrifugal concentrator $(3 \mathrm{kDa}$ cut off, GE Healthcare, Buckinghamshire, UK). The supernatant was collected as ES product. Protein concentrations were measured by a Bradford assay (Bio-Rad, Hercules, California) for soluble CWE and ES product, and by a BCA assay (Thermo Fisher Scientific) for insoluble CWE. All proteins were stored at $-20^{\circ} \mathrm{C}$ for $20 \mathrm{~min}$ prior to further experiments.

\section{Western blot analysis}

O. viverrini soluble and insoluble CWEs ( $20 \mu \mathrm{g}$ each), ES products $(20 \mu \mathrm{g})$ and rOvCaBPs (100 ng each) were size-separated by $12.5 \%$ SDS-PAGE and transferred onto a nitrocellulose membrane (Bio-Rad) by semi-dry transfer using a Fastblot B33 instrument (Whatman, Biometra, Germany). The membrane-bound proteins were probed separately with the 4 specific rOvCaBP antisera at a dilution of 1:2,000. Pre-immune sera were used as negative controls at the same dilution. Alkaline phosphatase-goat anti-mouse IgG (Sigma, Saint Louis, Missouri) was used as secondary antibody $(1: 30,000)$. Colorimetric signals were developed with BCIP/NBT phosphatase substrate (Amresco, Solon, Ohio, USA). Each recombinant OvCaBP was also detected with pooled O. viverrini- infected hamster sera $(\mathrm{n}=10)$ by Western blot analysis. The 4 rOvCaBPs (500 ng each) and soluble CWE $(5 \mu \mathrm{g})$ were size-separated by $12.5 \%$ SDS-PAGE and transferred onto a nitrocellulose membrane as described above. Pooled pre-infection sera were used as a negative control $(n=10)$. The membranes were incubated with 12-week-infected sera at a dilution of 1:200 or preinfection sera at the same dilution. Goat anti-hamster IgG conjugated HRP (Life technologies, Maryland) and Metal enhanced DAB (3,3'-diaminobenzidine) substrate kit (Thermo Scientific, Chicago, Illinois) were used as secondary antibody and colorimetric substrate, respectively.

\section{Immunohistochemistry}

The distribution of each OvCaBP in mature (8-week-old) parasites was analyzed in paraffin- embedded tissue-sections as described in our previous experiments (15). All mouse antirOvCaBP antisera and mouse pre-immune sera were used at a dilution of 1:1,000. A biotinylated rabbit anti-mouse antibody (Dako, Carpinteria, California) was used as secondary antibody (1:200). Colorimetric detection was performed by using an $\mathrm{ABC}$ peroxidase detection kit (Thermo Scientifi). The signals were developed using AEC (3-amino-9-ethylcarbazole) 
substrate (Thermo Fisher Scientific).

\section{Analysis of ion binding properties by mobility shift assay}

Native polyacrylamide gel electrophoresis without SDS was used to study the ion-binding properties of OvCaBPs by changes in their migration patterns. Five micrograms of each rOvCaBP and rGST (glutathione S-transferase, negative control, expressed by pGEX-5X-1, GE Healthcare, Illinois) was preincubated with $5 \mathrm{mM}$ EDTA to chelate contaminating metal ions. EDTA-treated proteins were incubated with $25 \mathrm{mM} \mathrm{CaCl}$ or without $\mathrm{CaCl}$. Electrophoresis buffer $(120 \mathrm{mM}$ Tris-HCl, $0.05 \%[\mathrm{w} / \mathrm{v}$ ] Bromothymol blue, 1\% [w/v] DTT, 20\% [v/v] glycerol) was then added to the samples and thoroughly mixed. The samples were resolved in $8.5-12.5 \%$ continuous native gels in SDS-free electrophoresis buffer. The recombinant proteins were visualized by Coomassie blue staining. In addition to $\mathrm{Ca}^{2+}$, the divalent cations $\mathrm{Mg}^{2+}, \mathrm{Zn}^{2+}$ and $\mathrm{Cu}^{2+}$ were also tested at the same concentration.

\section{REFERENCES}

1. Srisawangwong T, Sithithaworn P, Tesana S. Metacercariae isolated from cyprinoid fishes in Khon Kaen district by digestion technic. Southeast Asian J Trop Med Public Health 1997; 28: 224226.

2. Sriraj P, Aukkanimart R, Boonmars T, Wonkchalee N, Juasook A, Sudsarn P, Pairojkul C, Waraasawapati S, Pinlaor S. Alcohol and alkalosis enhance excystation of Opisthorchis viverrini metacercariae. Parasitol Res 2013; 112: 2397-2402.

3. Geadkaew A, von Bülow J, Beitz E, Tesana S, Vichasri Grams S, Grams R. Bi-functionality of Opisthorchis viverrini aquaporins. Biochimie 2015; 108: 149-159.

4. Young ND, Campbell BE, Hall RS, Jex AR, Cantacessi C, Laha T, Sohn WM, Sripa B, Loukas A, Brindley PJ, Gasser RB. Unlocking the transcriptomes of two carcinogenic parasites, Clonorchis sinensis and Opisthorchis viverrini. PLoS Negl Trop Dis 2010; 4: e719.

5. Wang X, Chen W, Huang Y, Sun J, Men J, Liu H, Luo F, Guo L, Lv X, Deng C, Zhou C, Fan Y, Li X, Huang L, Hu Y, Liang C, Hu X, $\mathrm{Xu}$ J, $\mathrm{Yu} \mathrm{X}$. The draft genome of the carcinogenic human liver fluke Clonorchis sinensis. Genome Biol 2011; 12: R107.

6. Huang Y, Chen W, Wang X, Liu H, Chen Y, Guo L, Luo F, Sun J, Mao Q, Liang P, Xie Z, Zhou C, Tian Y, Lv X, Huang L, Zhou J, Hu Y, Li R, Zhang F, Lei H, Li W, Hu X, Liang C, Xu J, Li X, Yu X. The carcinogenic liver fluke, Clonorchis sinensis: new assembly, reannotation and analysis of the genome and characterization of tissue transcriptomes. PLoS One 2013; 8: e54732.

7. Young ND, Nagarajan N, Lin SJ, Korhonen PK, Jex AR, Hall RS, Safavi-Hemami H, Kaewkong W, Bertrand D, Gao S, Seet Q,
Wongkham S, Teh BT, Wongkham C, Intapan PM, Maleewong W, Yang X, Hu M, Wang Z, Hofmann A, Sternberg PW, Tan P, Wang J, Gasser RB. The Opisthorchis viverrini genome provides insights into life in the bile duct. Nat Commun 2014; 5: 4378.

8. Rice P, Longden I, Bleasby A. EMBOSS: the European Molecular Biology Open Software Suite. Trends Genet 2000; 16: 276-277.

9. Huerta-Cepas J, Serra F, Bork P. ETE 3: Reconstruction, analysis, and visualization of phylogenomic data. Mol Biol Evol 2016; 33: $1635-1638$.

10. Sievers F, Wilm A, Dineen D, Gibson TJ, Karplus K, Li W, Lopez R, McWilliam H, Remmert M, Soding J, Thompson JD, Higgins DG. Fast, scalable generation of high-quality protein multiple sequence alignments using Clustal Omega. Mol Syst Biol 2011; 7: 539.

11. Edgar RC. MUSCLE: multiple sequence alignment with high accuracy and high throughput. Nucleic Acids Res 2004; 32: 17921797.

12. Katoh K, Kuma K, Toh H, Miyata T. MAFFT version 5: improvement in accuracy of multiple sequence alignment. Nucleic Acids Res 2005; 33: 511-518.

13. Wallace IM, O'Sullivan O, Higgins DG, Notredame C. M-Coffee: combining multiple sequence alignment methods with T-Coffee. Nucleic Acids Res 2006; 34: 1692-1699.

14. Capella-Gutiérrez S, Silla-Martínez JM, Gabaldón T. trimAl: a tool for automated alignment trimming in large-scale phylogenetic analyses. Bioinformatics 2009; 25: 1972-1973.

15. Guindon S, Dufayard JF, Lefort V, Anisimova M, Hordijk W, Gascuel $\mathrm{O}$. New algorithms and methods to estimate maximumlikelihood phylogenies: assessing the performance of PhyML 3.0. Syst Biol 2010; 59: 307-321.

\section{ADDITIONAL RESULTS AND DISCUSSION}

As described in the main publication the 4 anti-rOvCaBP antisera showed cross reactivity. This is also demonstrated in Supplementary Fig. 4. Therefore, the results of the immunohistochemical analysis are underdetermined, i.e. it cannot be excluded that the observed staining obtained with each antiserum originates from the detection of more than one isoform. A common problem if conserved isoforms have comparable expression profiles.

The analysis of paraffin-embedded tissue from adult $O$. viverrini with anti-rOvCaBP1-3 antisera detected the antigens in the tegument layer and at a lesser staining intensity in the tegumental cell bodies (Supplementary Fig. 5). OvCaBP4, surprisingly, could not be detected. Although the detection might not be isoform-specific it supports the largely tegumental distribution reported for other family members. Staining in the tegumental cell bodies was often weak or absent suggesting fast 
transport of the proteins to the syncytial tegument after translation in the cell bodies. Unexpectedly, anti-rOvCaBP4 antisera, while positive with CW extracts and ES product in western analysis, could not detect OvCaBP4 in the parasite tissue. We tried antibody dilutions as low as 1:100 and several epitope-retrieval conditions without success and concluded that the antisera from the 3 mice did not detect the set of epitopes available in the tissue sections. The orthologous CsTegu21.6 was detected in the tegument but seemingly weakly [1]. The results are also comparable with CsTegu20.6 [2]. In F. gigantica, FgCaBP1, 3 and 4 were found in the tegument, the epithelial lining of the excretory system, and in the intestinal tract $[3,4]$. OvCaBP22.8, as mentioned above, was detected in the intestinal tract and parenchyma, but not in the tegument [5]. This indicates that distinct family members can have roles in different tissue types and that it is important to analyze the basic properties of all family members.

\section{REFERENCES}

1. Subpipattana P, Grams R, Vichasri-Grams S. Analysis of a calcium-binding EF-hand protein family in Fasciola gigantica. Exp Parasitol 2012; 130: 364-373.

2. Kim YJ, Yoo WG, Lee MR, Kim DW, Lee WJ, Kang JM, Na BK, Ju JW. Identification and characterization of a novel 21.6-kDa tegumental protein from Clonorchis sinensis. Parasitol Res 2012; 110: 2061-2066.

3. Kim YJ, Yoo WG, Lee MR, Kang JM, Na BK, Cho SH, Park MY, Ju JW. Molecular and structural characterization of the tegumental 20.6-kDa protein in Clonorchis sinensis as a potential druggable target. Int J Mol Sci 2017; 18: 557.

4. Vichasri-Grams S, Subpipattana P, Sobhon P, Viyanant V, Grams R. An analysis of the calcium-binding protein 1 of Fasciola gigantica with a comparison to its homologs in the phylum Platyhelminthes. Mol Biochem Parasitol 2006; 146: 10-23.

5. Senawong G, Laha T, Loukas A, Brindley PJ, Sripa B. Cloning, expression, and characterization of a novel Opisthorchis viverrini calcium-binding EF-hand protein. Parasitol Int 2012; 61: 94-100. 


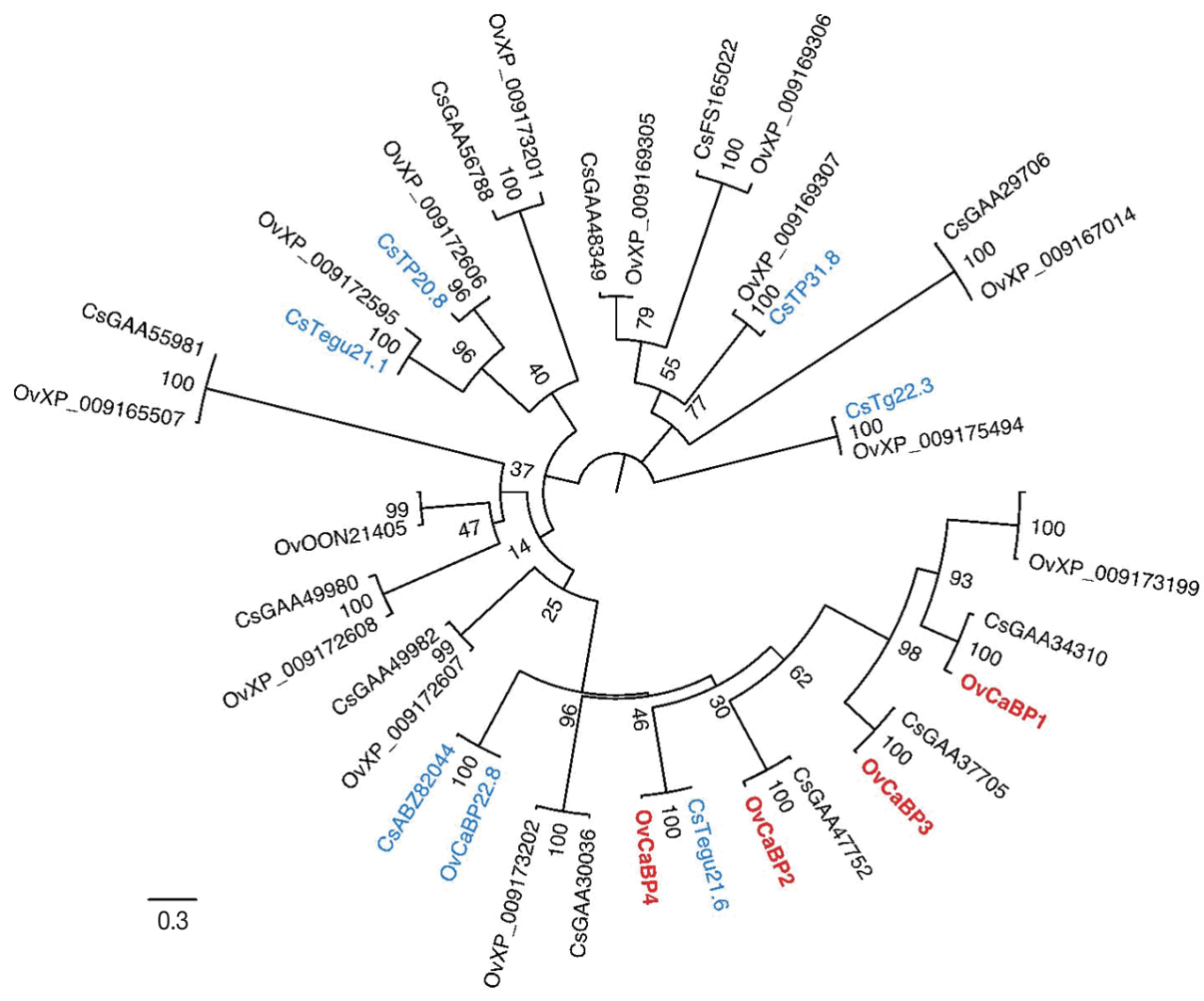

Supplementary Fig. 1. Phylogenetic tree based on maximum likelihood analysis of 19 O. viverrini/C. sinensis CaBPs. The described OvCaBP1-4 are indicated in red color and previously analyzed C. sinensis and O. viverrini proteins in blue color (CsTegu21.6, AEl69651; OvCaBP22.8, XP_009173200; CsABZ82044, ABZ82044; CsTg22.3, ABK60085; CsTP31.8, ABK60086; CsTegu20.6, GAA49981; CsTP20.8, ABC47326; CsTegu21.1, ADZ13689). Uncharacterized proteins are named based on their accession numbers. The bootstrap support values are shown at the nodes, this is an unrooted tree, log likelihood: -7,368.243964. 

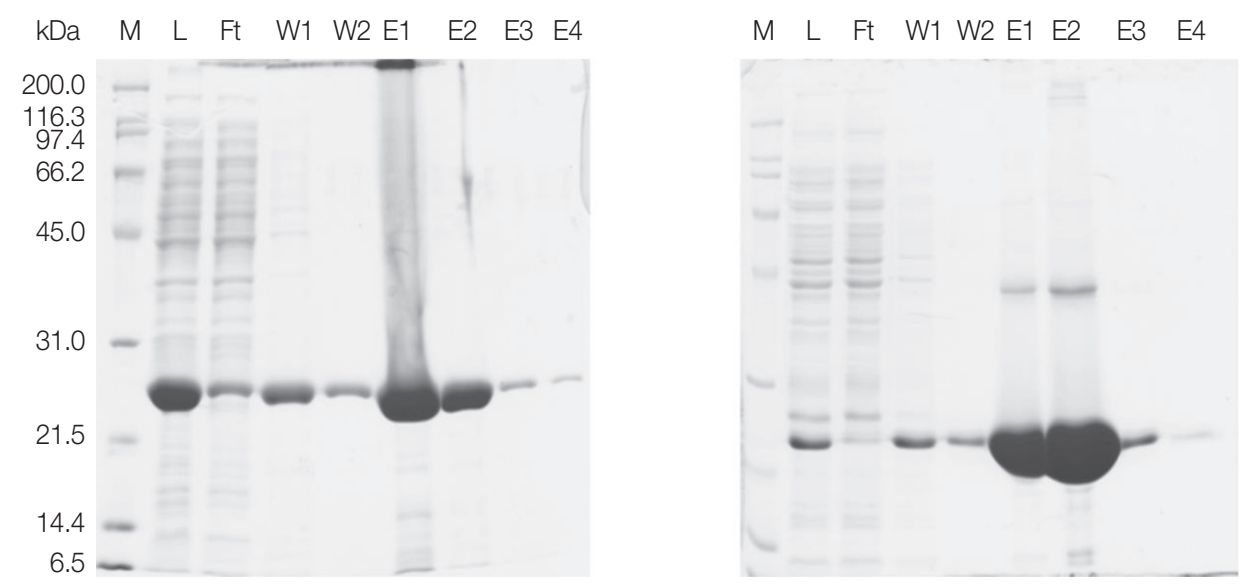

$\begin{array}{lllllll}k D a & F t & \text { W1 } & \text { E1 } & \text { E2 } & \text { E3 } & \text { E4 }\end{array}$

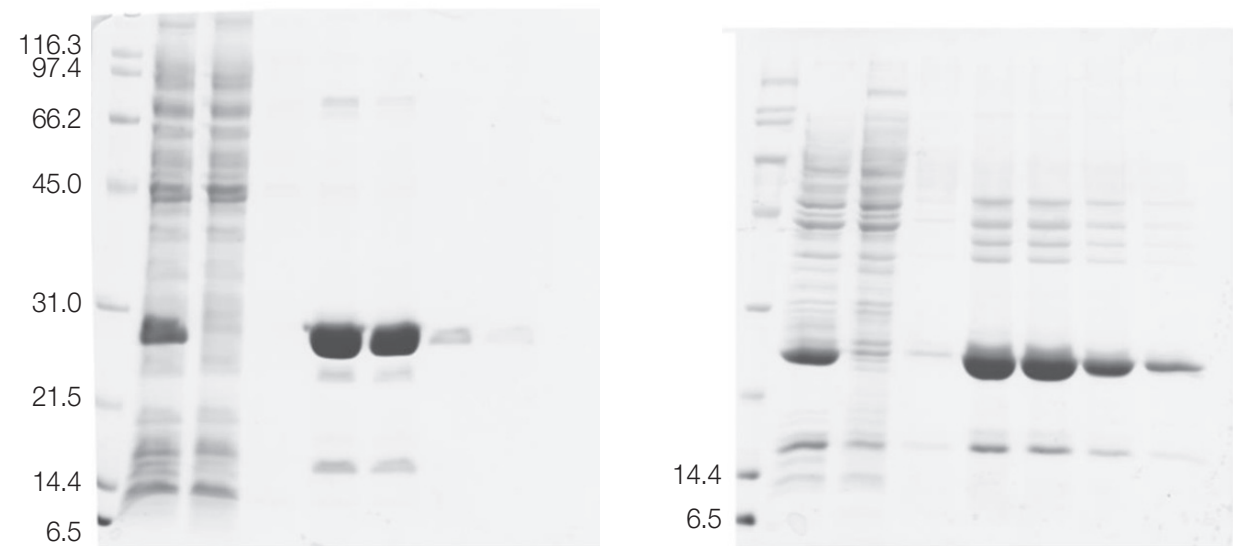

Supplementary Fig. 2. Purification of recombinant OvCaBPs under native conditions. Collected fractions were resolved by $12.5 \%$ SDSPAGE including bacterial lysate (L), flow-through fraction (Ft), wash fractions (W1, W2) and elution fractions (E1-4). Each rOvCaBP was found highly purified at the expected mass in E1 and E2 fractions. Lane M: broad range protein standard marker (Bio-Rad). 


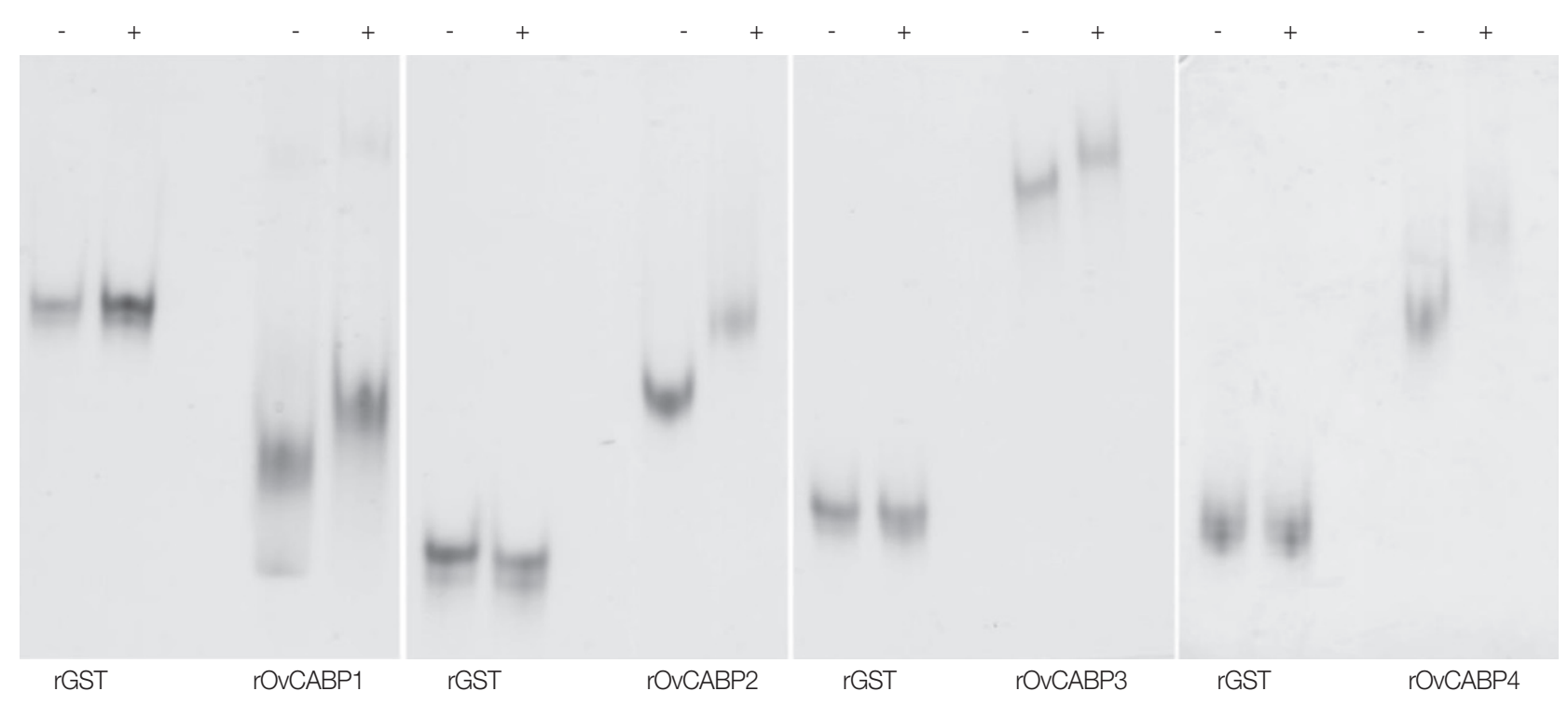

Supplementary Fig. 3. Determination of calcium-binding property by gel mobility shift assay (8.5 or $12.5 \%$ non-denaturing gel). Five micrograms of the recombinant protein was pre-incubated with 5 mM EDTA and post-incubated with 25 mM CaCl while rSjGST was used as a negative control. Presence and absence of $\mathrm{CaCl}$ are indicated by plus (+) and minus (-) symbols, respectively. 


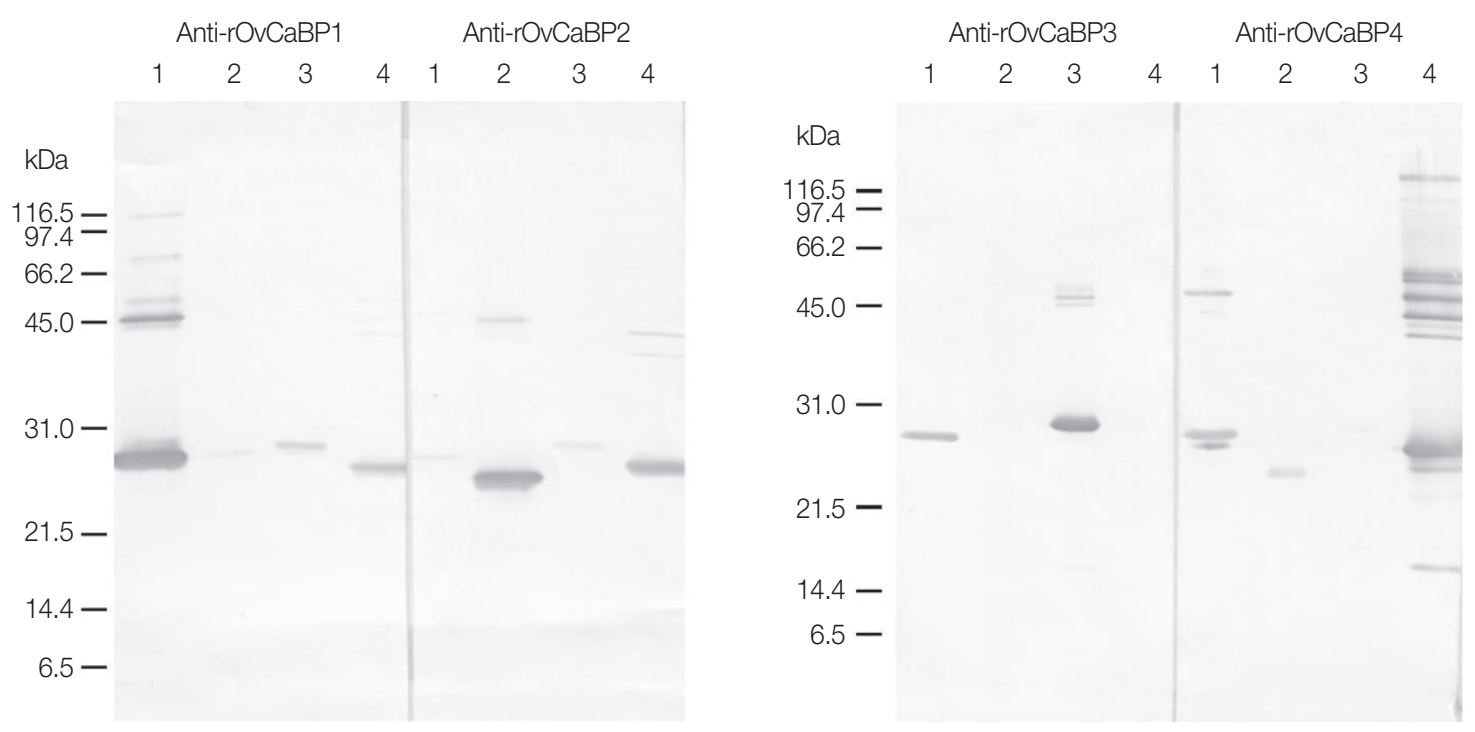

Supplementary Fig. 4. Western analysis of cross-reactivity of mouse anti-rOvCaBP antisera. Each rOvCaBP was independently probed with each of the four anti-rOvCaBP antisera at a dilution of 1:2,000. Lanes 1-4: 100 ng of rOvCaBP1, rOvCaBP2, rOvCaBP3 and rOv$\mathrm{CaBP} 4$, respectively. Protein standard sizes are indicated on the left. 

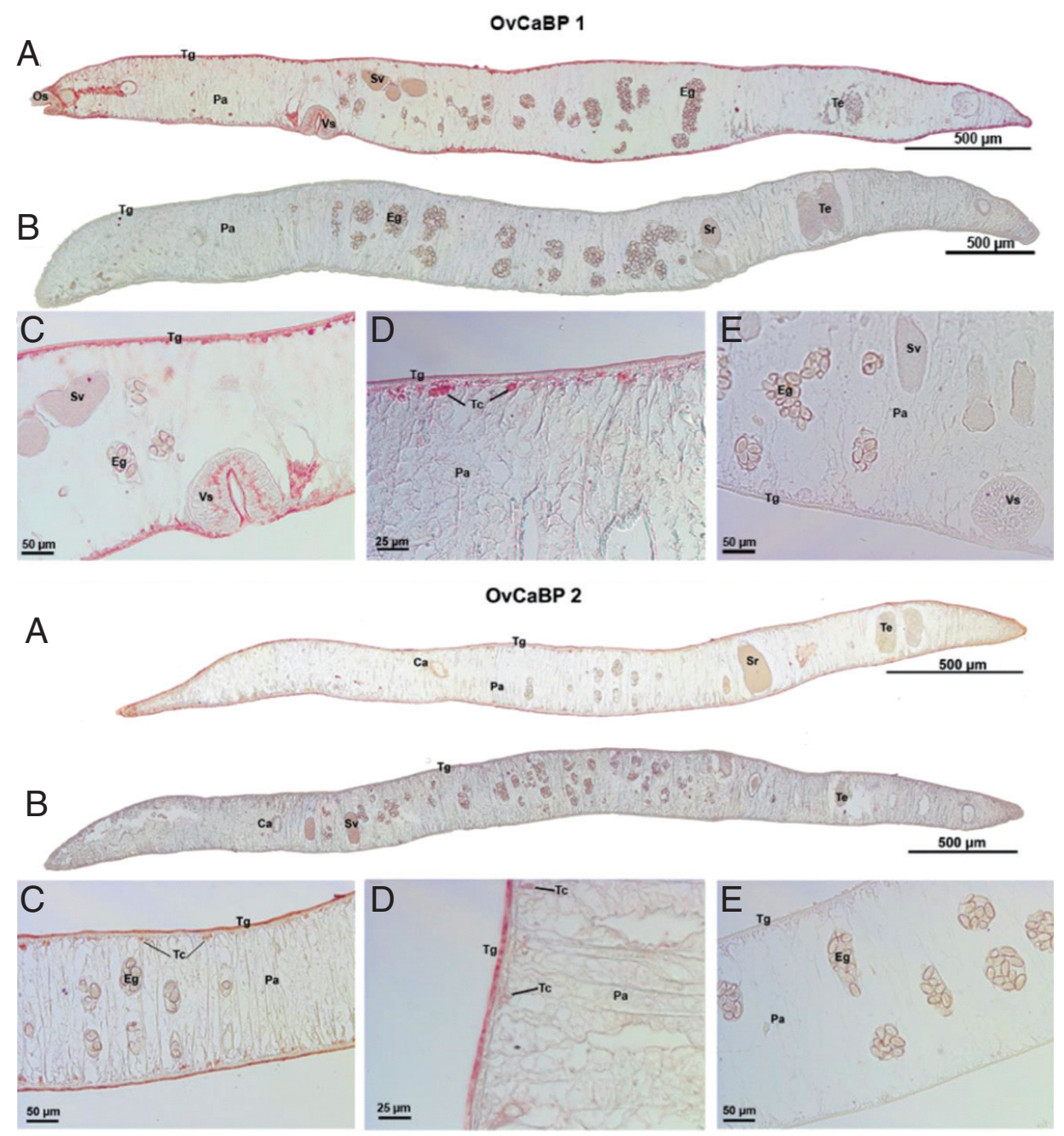

OvCaBP 3

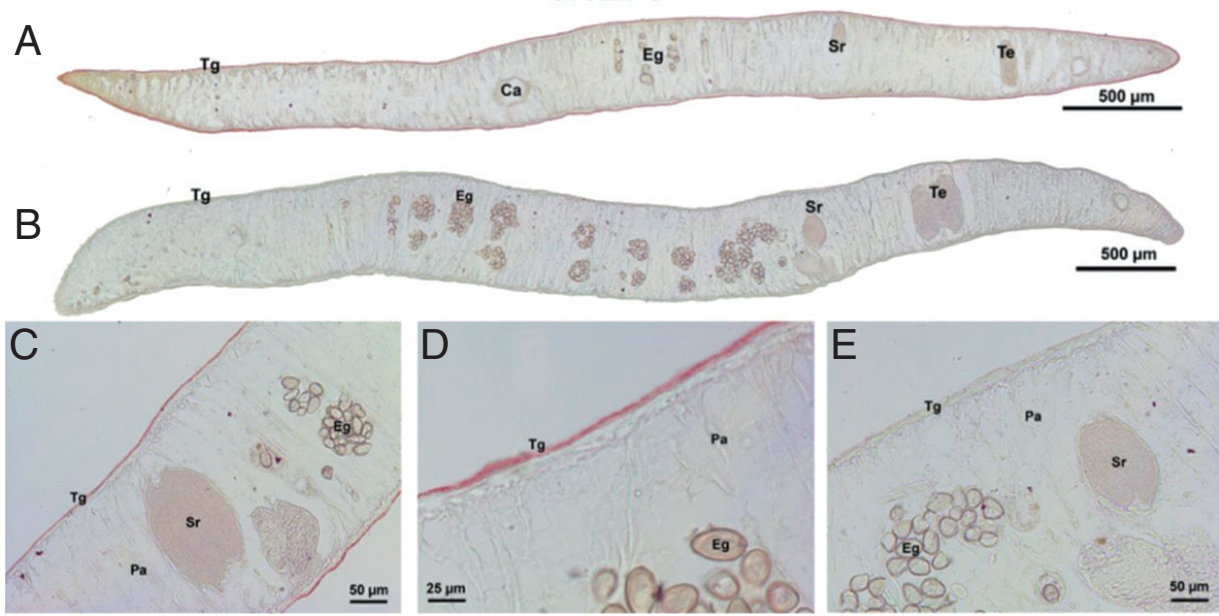

Supplementary Fig. 5. Immunohistochemical detection of OvCaBPs in adult O. viverrini tissue by mouse anti-rOvCaBP antisera at dilution 1:1,000. Positive red staining is present in tegument and tegumental cell bodies (OvCaBP1 and OvCaBP2) of the parasite (A, C, and D). No staining was observed in the tissue-sections detected with pre-immune sera (B and E). Ca, cecum; Eg, egg; Os, oral sucker; $\mathrm{Pa}$, parenchyma; Sr, seminal receptacle; Sv, seminal vesicle; Tc, tegumental cell bodies; Te, Testis; Tg, tegument; Vs, ventral sucker. 